give brief accounts of research projects covering a wide field including stroke, head injury, brain tumours and brain death. Basic haemodynamics as well as practical applications such as intraoperative monitoring are dealt with.

The volume is very much one for the specialist working in the field. It enables him or her to see what work is going on and the results being obtained. It would not be of interest to the general reader.

J MARSHALL

The Threatened Medical Identity of Psychiatry: The Winds of Change.

By THEODORE PEARLMAN. (Pp 270; Price: \$49.75) 1992 Illinois USA, Charles $C$ Thomas. ISBN 0-398-05768-0

The author grew up in South Africa and worked there as a general practitioner before specialising in psychiatry and emigrating to the United States. He has written a series of essays on the present practice of psychiatry principally in the country of his adoption. These range from a critique of DSM3 (a USA classification of psychiatric disorders) to excursions into psychopolitics (Russian and South African psychiatry).

The tone is informal, loosely structured and highly personal. Idiosyncratic assertions and dogmatic opinions abound. Dr Pearlman agonises over the state of psychiatry and diagnoses an identity crisis. He deplores the medicalisation of behavioural disorders and is equally scathing about the claims of dynamic psychotherapy and biological psychiatric research. Psychopharmacology fares little better. In his scheme of things the psychiatrist is a hospital based general physician with an interest in psychological disorders, at the ready to diagnose organic aetiologies underlying psychiatric syndromes and to advise on adverse psychological reactions to physical disease. He has little to say about the chronic psychoses other than a half-approving nod in the direction of Lang and Szasz. The practice of psychiatry in the community hardly merits a mention. $\mathrm{He}$ is rather better on some of the more bread and butter issues that face American psychiatry: the influence of health insurance on the quality of care; the territorial battles between psychiatrists and psychologists; and the increasing bureacratisation of medicine. This is very much one man's view of his own speciality and should be treated as such

Problems and Methods in Longitudinal Research: Stability and Change. Edited by: D MAGNUSSON, L R BERGMAN, G RUDINGER and B TÖESTAD (Pp 351; Price $\mathrm{H} / \mathrm{b} £ 55.00$; $\$ 100.00)$. 1991. Cambridge, University Press. ISBN 0-521-40195-X

This is a collection of papers which were read at a workshop organized by the European Network on Longitudinal Studies on Individual Development (ENLS) within the framework of the European Science Foundation. The book is the fifth of a series of volumes dealing with various methodological problems in longitudinal research; the present one concentrating on problems of statistical analysis and interpretation of data. In particular, the aim of the present volume is to illustrate an indispensible link between the correct method of data treatment and the explicit formulation of a substantive problem. With this aim, each chapter (written by different authors) sets out a particular problem and describes a particular approach to the solution of the problem. Presumably, in the workshop itself, there might have been discussion concerning alternative strategies for solving these particular problems, stressing relative strengths and weaknesses of the various statistical methodologies. This is completely missing from the book, however, and for this reason the book is rather unsatisfactory from the reviewer's point of view. There is very little link between the chapters and very little evidence of communication between the authors. For this reason the beginner in this area will not find this volume as useful as a well-written textbook. It is a book to dip into to see how a particular worker proposes to solve some particular challenge.

There are fifteen chapers. I found the later chapters the more interesting, starting with Chapter 7, by Pickles and Rutter, in which they discuss searches for 'turning points' in developmental processes. The chapter on the use of event history models in social mobility research (Blossfeld, Hamerle and Mayer) was also very useful. There were two chapters on modelling genetic effects in longitudinal research, but these are perhaps a little specialised for the general reader. Of more general interest is the chapter on structural equation modelling of intellectual development (Rudinger, Andres and Rietz) and one on latent structure models for discrete data (Anderson)

I do not think that I learnt a lot from scanning through the pages of this volume, however, except in the chapter by Shröder, Edelstein and Hoppe-Graff on qualitative analyses of individual differences in intraindividual change.

GRAHAM DUNN

\section{Current Trends in the Treatment of Par-} kinson's Disease

(Proceedings of the symposium on Current Trends in the Treatment of Parkinson's Disease held in Venice on 3-5th May 1991) Edited by yves aGID (Pp 106; Price $£ 18.00$ ) 1992. London, John Libbey \& Co Ltd. ISBN 0861963393

This volume is the proceedings of the symposium held in 1991 to coincide with the European launch of Pergolide. The introduction suggests that the book will help clinicians make up their minds on the use of L-dopa and dopamine agonists in the treatment of Parkinson's disease. There is, however, little new data, but significant lobbying designed to help anyone take sides on important issues such as is L-dopa really toxic and does its 'early use accelerate development of fluctuations? and the reverse: do agonists with or without some L-dopa, delay such complications or really have any neuroprotective qualities? L-dopa preparations have provided the staple treatment world-wide for two decades improving both quality and quantity of life. Perhaps we need a Dopa appreciation society to ensure that it is not found guilty before we have convincing evidence, particularly as polypharmacy has its intrinsic problems and the very long term effects of agonist therapy are not yet known.
Although disappointing in its proposed aims there are some worthwhile and informative chapters. Within the first section on cell mortality mechanisms, the group from Pisa gives an interesting review on MPTP toxicity and the possible involvement of excitatory amino acids, including some provocative comments questioning the importance of the mitochondrial defect, at least as an explanation for cell death. The next chapter brings up the question of dopamine neurotoxicity involving some well known proposed mechanisms though with little evidence that they are active in vivo, except under conditions of ischaemia. It is followed by a nice review by Olanow of the role of free radicals in Parkinson's disease and how they could interact with other mechanisms such as excitotoxic and mitochondrial defects. Walters reviews current therapeutic approaches and the theoretical background to both palliative and protective treatment, followed by Tolosa on current strategies for L-dopa, administration with comments on the unproven idea that controlled release formulations of L-dopa could be less toxic than conventional preparations. There are additional chapters describing the molecular biology of dopamine receptors and Lees reviews the current indications for apomorphine and possible future directions The book finishes with two chapters giving a summary of the clinical data on Pergolide and some practical experience from both Milan and Houston.

Although this book does not come up to its stated aims there are a number of well written and interesting chapters from leaders in the field.

A C WILLIAMS

Disorders of the Cervical Spine. Edited by: $M$ B CAMINS and $P$ F O'LEARY (Pp 628 Illustrated; Price: $£ 115.00)$. 1992. London, Williams \& Wilkins Ltd. ISBN 0-683-01401-3

This is a large volume devoted to all aspects of diseases of the cervical spine, the contributors being mainly neurosurgeons and orthopaedic surgeons. It is not, however, unduly biased towards detailed descriptions of operative techniques and starts with good discussions with biomechanics, clinical syndromes, radiological investigations and neurophysiological monitoring. The scope of later chapters is wide enough to include enigmatic conditions such as thoracic outlet syndrome, cervical angina, and dysphagia and vertebral artery compression attributed to spondylotic spurs.

The section on trauma is notably good and includes excellent chapters on the classification of injuries of the cervical spine, basic principles of treatment and the use of Halo vests in management. There is much of interest in other sections including a good account, from Japan, of ossification of the posterior common ligament and on the use of decompressive laminoplasty.

Though the editors have chosen their subjects and authors so as to provide a very comprehensive account of the cervical spine, they have managed to avoid, on the whole, overlap between chapters. References are variable both in quantity and in up to datedness. The reviewer was unable to find any reference to very high doses of methyl prednisolone now used in spinal injury. There 
is also a lack of uniformity in the way the references are organised, some being presented in the old and much more useful alphabetical order and others are listed under the order in which they are quoted, a regrettable modern practice. The chapters are clearly written though not free from typographical errors and malapropisms (if such can be said to exist in an era of descriptive lexicography) and the reader may suffer from excessive exposure to acronyms. Even spinal cord injury is dealt with in this way.

Generally the book can be recommended as a reference text for departments and individuals.

TT KING

Bailliere's clinical neurology. International Practice and Research. (Vol. 1/No. 1 April 1992-Neurological Aspects of Human Retroviruses). Guest Editor: P RUDGE (Pp 262; Price: $£ 27.50)$. 1992. London, Bailliere Tindall. ISBN 0-7020-1629-2.

This multiple author book aims to summarise what is known about retroviral involvement of the nervous system in man. The first two chapters consist of a comprehensive but rather indigestible discourse on the classification, molecular biology and immunology of the known retroviral diseases. There is a further chapter on retroviral diseases in animals. I felt that for a book aimed at a clinical audience these were rather too long and could have been condensed.

An excellent chapter, describing the neurological syndromes associated with human $T$ cell leukaemia virus infection, follows. The remainder of the book is devoted to human immunodeficiency virus (HIV) infection, beginning with a useful brief overview of the associated disease spectrum. Chapters on opportunistic infections, cognitive impairment and other neurological disorders associated with HIV infection come next. Finally there is a section on pathology and a review of overall management.

It is interesting to see a European book on this subject. Although HIV infection produces broadly similar features in all Western cultures there are clearly subtle differences on this side of the Atlantic, for example in the relative frequencies of the different opportunistic infections. There is a certain amount of repetition in some of the early chapters but overall I found this a good book that achieves its aims. It would be a useful addition to any departmental library.

TJ WALLS

London Neurogenetics Database. M BARAITSER and R M wINTER (Price: $£^{395.00)}$. Oxford University Press. 1991. ISBN 0-19-262-039-8

Many Doctors are faced with the necessity of counselling patients or their relatives concerning hereditary disease. To do this requires confidence and detailed information. This electronic information resource provides both.
The greater part of it is a table of recog nised neurogenetic syndromes (about a thousand), with synonyms. This table can be browsed either by name (e.g., "Which syndromes contain the word CLEFT in their name?") or, more usefully, by clinical features. By trial, and with selection of features by their significance, it is possible to establish a list of syndromes that match a clinical picture, of a manageable size. To do this task by hand would be possible: it is in principle little different from selecting a particular bundle of groceries from a supermarket. It is beyond this point that the value of the electronic technique is seen, because, effortlessly, the database can display further information about the selected syndromes. It will provide an abstract of each syndrome and references to the original papers. This is the information that is needed to prune the list of syndromes individually to those few which are the possible diagnoses in a particular case. The database also allows searching of the reference list alone and this is in itself a valuable resource because there are just over ten thousand references. There is a facility to file data referring to patients in a third database (which can be stored off the computer). This is particularly useful in those situations when a diagnosis cannot be reached initially, and one needs to allow time to elapse as a diagnostic test-either to allow the significance of particular features to become apparent or because important features are age-related.

There are other methods such as working from the genetic defect where known. Whilst no one would claim to be pan-optic, there is a good case for feeling that if a tool like this does not turn up a recognised hereditary cause for a syndrome (however genetic it may look) then there is no such cause. The user's guide warns that this is a system for experts rather than an expert system as such. This database has many strengths: the instructions on how to install it give the correct information on hardware and software requirements, though finding the 12 MByte of disk space required to run it might be difficult. The instructions are easy to follow. The database is small enough to fit on a reasonable number of floppy disks rather than a CD-ROM. Updates and supplements are planned.

The techniques and procedures needed to operate the database are clearly described in the well-produced manual, and easy to master. There are some points that the prospective user should be aware of. It is plainly intended for the practising clinician and will be of far greater use in the consulting room than in the library. It is strongly slanted towards paediatric practice. Apart from the database's being the product of many years' work from one unit, its organisation represents a fairly individualistic way of going about things. Not many Doctors would wish to keep patient records (even in abstracted form) on a computer.

The overall value of the data is outstanding, and the high quality of the software and presentation (such as the user's guide and manuals) is what one would expect from a major publishing house. There is a commitment to extending this work, and keeping it up-to-date. The main audience for this database is self-defining, but it is to he hoped that many neurologically-inclined Doctors will look at it, as it replaces many paper-based resources and, therefore, makes available information otherwise inaccessible due to constraints of time.

BP FOWLER
Treatment of Multiple Sclerosis Trial Design, Results and Future Perspectives. Edited by: R A RUDICK and D E GOODKIN (Pp 313; Price: DM 210.00). 1992. Heidelberg, Springer-Verlag. ISBN 3-540-19683-8

This book bears testimony to the difficulties of assessing treatment in such a variable disease over the years by devoting almost hal its content to an exhaustive and masterly review of the methodology, and its pitfalls. This section is thus more suited to those who might design future trial protocols than to the clinician who dips into its pages for an update on current treatment options. Indeed the ain title may mislead-this is not a comprehensive text of treatment in MS but rather critical appraisal of attempts to alter or slow the progression of the disease, focussing on specific immunotherapies. Nevertheless at time of rapid expansion in immunology book with such a perspective is timely. Many Neurologists without immunological expertse will welcome this comprehensive critique of immunotherapy in this common condition.

The second half of the book relates to specific forms of immunotherapy and comprises comprehensive reviews of treatmen data to date often enhanced by the authors' personal experience of their 'pet treatment' Other chapters are presented in the format of an extended paper which is perhaps less objective. The chapters on Natural History (Goodkin) and Steroid Treatment (Myers) were particularly enjoyable.

The final two chapters provide an enticing insight into future therapy, aimed at mor specific (but as yet hypothetical) methods of immune intervention. If these aspirations are realised, the meat of the current text may become relegated to the earlier historical chapters. This book will earn its place on the shelf of those who wish to embark on the thorny road of evaluating modern treatments of MS rather than the general clinician.

DA FRANCIS

Functional Anatomy of the Neuroendocrine Hypothalamus (Ciba Foundation Symposium 168). Edited by D J CHADWICK (ORGANISER) AND J MARSH (Pp 300; Price 42.50). 1992. Chichester, J Wiley \& Son Ltd. ISBN 0-471-93440-2.

Clinical Neuroanatomy for Medical Students 3rd Edition. By $\mathrm{R} S$ SNELL (Pp 653 Illus: Micrographs, some colour; Price f24.95). 1992. Edinburgh, Churchill Livingstone. ISBN 0-316-80244-1.

Management of Acute Pain: A Practical Guide. Prepared and Edited by International Association for the Study of Pain (Tas Force on Acute Pain) Edited by L B READY and w T EDWARDS (Pp 73; Price US \$15.00) 1992 Seattle, IASP Publications. ISBN 0-931092 01-9. 